\title{
Preparation of graphene bilayers on platinum by sequential chemical vapour deposition
}

\author{
Johannes Halle, ${ }^{*}$ Alexander Mehler, Nicolas Néel, Jörg Kröger \\ Institut für Physik, Technische Universität Ilmenau, D-98693 Ilmenau, Germany \\ E-mail: johannes.halle@tu-ilmenau.de
}

\begin{abstract}
A cheap and flexible method is introduced that enables the epitaxial growth of bilayer graphene on $\mathrm{Pt}(111)$ by sequential chemical vapour deposition. Extended regions of two stacked graphene sheets are obtained by, first, the thermal decomposition of ethylene and the subsequent formation of graphene. In the second step, a sufficiently thick Pt film buries the first graphene layer and acts as a platform for the fabrication of the second graphene layer in the third step. A final annealing process then leads to the diffusion of the first graphene sheet to the surface until the bilayer stacking with the second sheet is accomplished. Scanning tunnelling microscopy unravels the successful growth of bilayer graphene and elucidates the origin of moiré patterns.
\end{abstract}

\section{Introduction}

Along with the ongoing interest in graphene and its derivatives comes the demand for reliable and scalable preparation methods for the eventual application in devices. For graphenebased layered systems such as bilayer graphene (BLG), whose properties depend strongly on stacking and interlayer twist angles, ${ }^{1,2}$ the epitaxial growth directly on the sample yielded high structural quality.

$\mathrm{SiC}$ substrates $^{3-5}$ and transition metal surfaces ${ }^{6,7}$ have widely been used for the preparation of monolayer graphene (MLG) as well as multilayer stackings. For the fabrication of closed monolayers on metals, the thermal decomposition of hydrocarbons assisted by the catalytically active surface is typically performed as a chemical vapour deposition (CVD). ${ }^{8}$ The growth is self-limiting to one monolayer and, thus, offers precise thickness control. Additionally, this technique yields high-quality sheets with only a few rotational domains depending on substrate and growth temperature ${ }^{7,9,10}$ and may readily be applied in industrial production. However, due to the self-limitation, its use for the preparation of multilayers is not straightforward. Instead, the growth of MLG and multilayer graphene on metals benefits from an alternative approach, namely the segregation of $\mathrm{C}$ at elevated temperatures. To this end, the bulk metal is artificially enriched with $\mathrm{C}$, either via doping from the gas phase of hydrocarbons $^{6,7,11-13}$ or solid-state diffusion. ${ }^{14-17}$ Alternatively, high-quality multilayer graphene 
on metals may also be achieved by intercalation of $\mathrm{C}$ under MLG, which requires sources of atomic C. ${ }^{18-20}$

A preparation protocol that enables the growth of homogeneous as well as heterogeneous stackings of two-dimensional materials in a controlled surface science approach would be highly desirable. Ideally, a CVD-based layer-by-layer preparation would combine the synthesis of large-scale graphene sheets with high structural quality and with precise thickness control owing to the self-limitation to one monolayer per growth cycle.

Here, we present a sequential CVD method that achieves the high-quality preparation of extended BLG regions on $\mathrm{Pt}(111)$. In a four-step process, MLG is first prepared on $\mathrm{Pt}(111)$ via thermal decomposition of $\mathrm{C}_{2} \mathrm{H}_{4}$, followed by the deposition of a thick $\mathrm{Pt}$ film and subsequent fabrication of a second graphene layer. During the postannealing in the last step, the diffusion of buried MLG to the surface is completed and leads to the formation of extended BLG domains. This last step can also be viewed as the intercalation of the deposited thick metal layer. Various materials were previously demonstrated to intercalate graphene, e.g., $\mathrm{H},{ }^{21} \mathrm{Au},{ }^{22,23} \mathrm{Co},{ }^{24,25} \mathrm{Fe},{ }^{26} \mathrm{Ni},{ }^{26,27} \mathrm{Ag},{ }^{28} \mathrm{Eu},{ }^{29} \mathrm{Cs},{ }^{29,30}$ as well as Li. ${ }^{31}$ Therefore, Pt is very likely to intercalate, too. A method akin to the one suggested here has recently been applied to prepare MLG flakes on $\mathrm{Au}(111) .{ }^{23}$ Since Au does not offer the convenient CVD synthesis, MLG flakes grown by CVD on $\operatorname{Ir}(111)$ were subsequently intercalated by several monolayers of $\mathrm{Au}$ to achieve graphene flakes on top of a $\mathrm{Au}(111)$ surface. All prepared graphene layers are thoroughly characterised by atomically resolved scanning tunnelling microscopy (STM).

\section{Results and discussion}

While in principle the presented preparation protocol should be applicable to $\mathrm{Ir}, \mathrm{Ru}, \mathrm{Ni}, 6,7$ single-crystalline (111) surfaces of Pt have been chosen in this work since on this substrate the segregation after a CVD process is typically insufficient to already produce BLG. Moreover, graphene on $\mathrm{Pt}(111)$ has the appealing property of the lowest graphene-metal coupling. ${ }^{32}$

Figure 1 illustrates the preparation process by STM images (Fig. 1a-d) and sketches (Fig. 1e-h). First, MLG results from epitaxial growth of thermally decomposed $\mathrm{C}_{2} \mathrm{H}_{4}$ (Fig. 1a,e). In most regions, moiré patterns due to the lattice mismatch between graphene and $\mathrm{Pt}(111)$ were absent in STM images, which hints at large rotation angles of graphene with respect to $\operatorname{Pt}(111) .{ }^{33,34}$ Occasionally, moiré superstructures were observed and indicated smaller rotation angles. ${ }^{35}$ Second, approximately 45 atomic layers of Pt were deposited on MLG-covered Pt(111). STM images show resulting Pt clusters (Fig. 1b,f). In the third step, the second layer of graphene was grown by thermal decomposition of $\mathrm{C}_{2} \mathrm{H}_{4}$ on the $\mathrm{Pt}$ film. The required annealing of the sample at $1000 \mathrm{~K}$ led to the incomplete diffusion of the first graphene layer to the surface giving rise to large MLG and many small BLG regions (Fig. 1c,g). Further evidence that the buried graphene sheet forms the bottom layer of BLG in this preparation method is provided in the SI. The intercalation of Pt under MLG due to the high temperature during the CVD possibly breaks the MLG apart. Indeed, domain boundaries of MLG assist the intercalation process ${ }^{30,36}$ and may likewise facilitate the rupture of MLG. ${ }^{37}$ Individual patches can then diffuse separately to the surface (Fig. 1g) as observed during the 


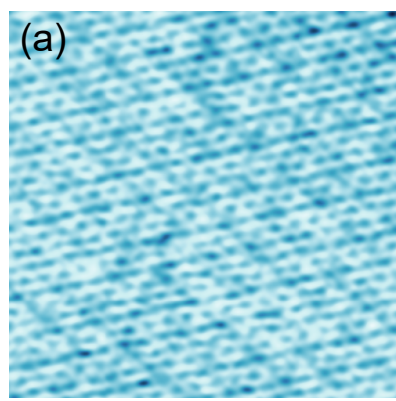

(e)

MLG

Pt substrate

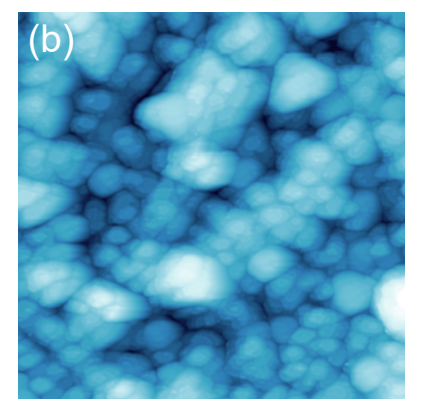

(f)

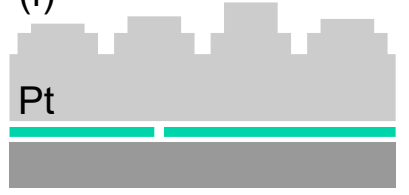

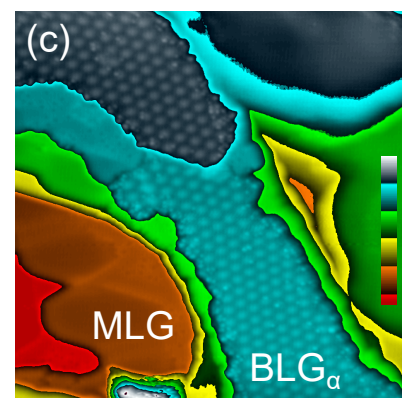

(g)

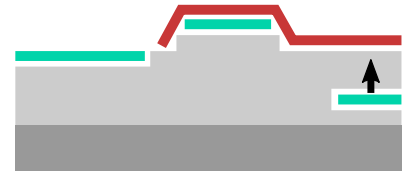

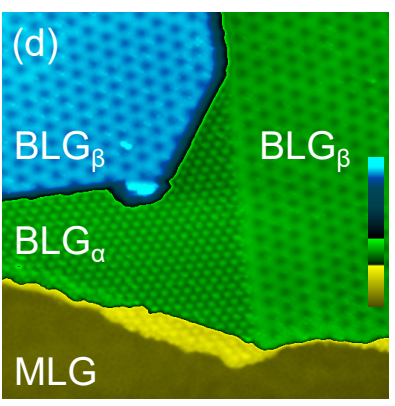

(h)

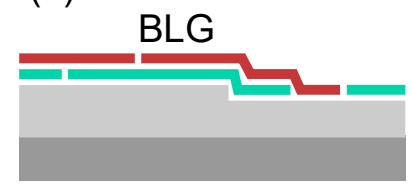

Figure 1: Steps of the BLG preparation protocol. (a) Atomically resolved STM image of MLG on Pt(111) (bias voltage: $V=74 \mathrm{mV}$, tunnelling current: $I=100 \mathrm{pA}$, size: $5 \times 5 \mathrm{~nm}^{2}$ ). (b) STM image of Pt film deposited on MLG/Pt(111) $\left(1 \mathrm{~V}, 80 \mathrm{pA}, 120 \times 120 \mathrm{~nm}^{2}\right)$. (c) STM image of MLG and BLG regions after the second CVD step $\left(1 \mathrm{~V}, 80 \mathrm{pA}, 40 \times 40 \mathrm{~nm}^{2}\right)$. (d) STM image of MLG and BLG after annealing the sample of (c) $\left(1 \mathrm{~V}, 90 \mathrm{pA}, 60 \times 60 \mathrm{~nm}^{2}\right)$. (e)-(h) Illustration (cross-section) of the preparation steps.

preparation of graphene nanoflakes on $\mathrm{Au}(111) / \operatorname{Ir}(111) .{ }^{23}$ Additionally, individual $\mathrm{C}$ atoms may detach from the boundary of buried graphene layers, segregate and contribute to the graphene growth at the surface. ${ }^{14-17}$ The third step clearly demonstrates that a sufficiently thick Pt film is crucial to avoid completion of the Pt intercalation before the actual growth of the second graphene layer can start. Subsequent annealing of the sample yielded flat and extended MLG and BLG regions (Fig. 1d,h). Second-layer graphene flakes hybridise to form extended BLG regions, while buried graphene patches continue their diffusion to the surface and may either be incorporated in the bottom graphene layer or create additional BLG or even trilayer graphene (TLG) regions.

Evidence for the proposed growth is given by the analysis of spatial periods $(\delta)$ and the orientations of the moiré pattern $(\varphi)$ and of the observed graphene lattice $(\vartheta)$ with respect to $\mathrm{Pt}(111)$. The details of the analysis are summarised in the SI. Moiré domains that do not match the expected characteristics of MLG are attributed to BLG.

Figure 2 summarises this analysis. MLG after the first step of the preparation protocol (Fig. 2a,b) occasionally exhibits moiré patterns with, e.g., $\delta=2.37 \mathrm{~nm}, \vartheta=4.8^{\circ}$ (Fig. 2a) and $\delta=0.82 \mathrm{~nm}, \vartheta=16.7^{\circ}$ (Fig. $2 \mathrm{~b}$ ). After the complete preparation moiré patterns of MLG with $\delta=0.79 \mathrm{~nm}, \vartheta=-15.8^{\circ}$ (Fig. 2c) are observed. The dark depressions, which most clearly appear in the STM image of Fig. 2c, may be due to $\mathrm{C}$ atoms in the Pt surface ${ }^{17}$ or vacancies. ${ }^{38}$ Figure $2 \mathrm{~d}$ shows an STM image where BLG and TLG are present as adjacent regions. The dashed line marks the boundary between the different domains. All moiré spatial periods and orientations (symbols) together with the expected trends for MLG (solid 

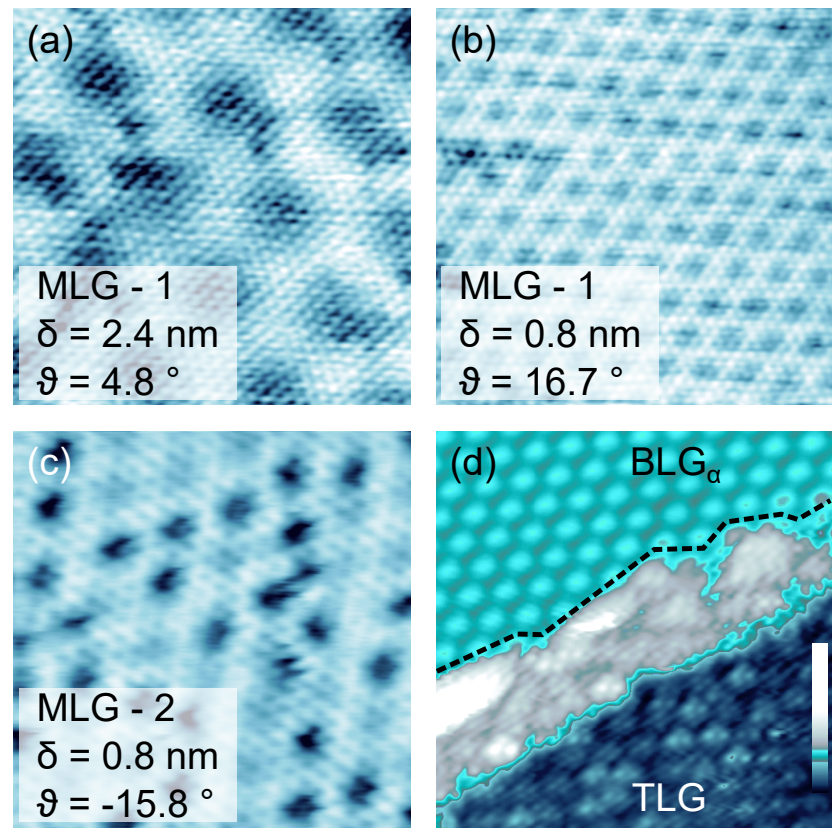

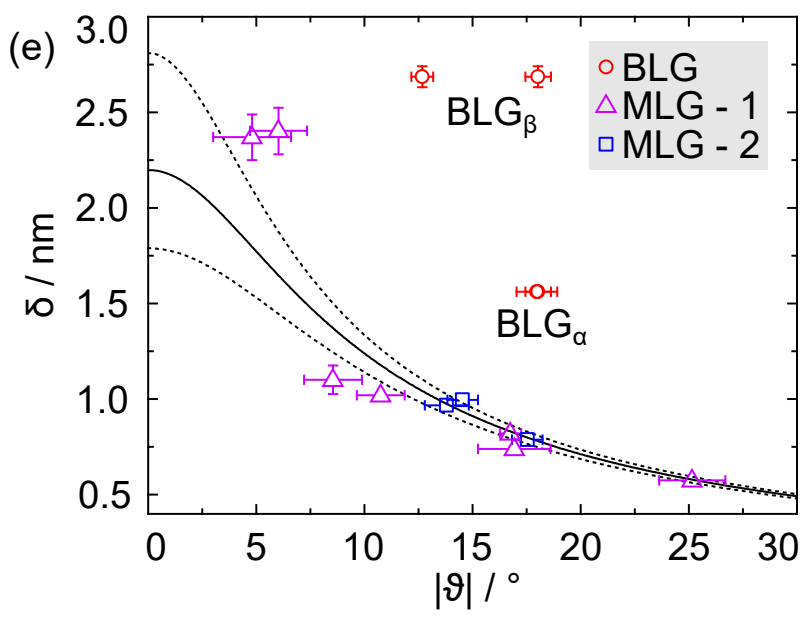

(f)

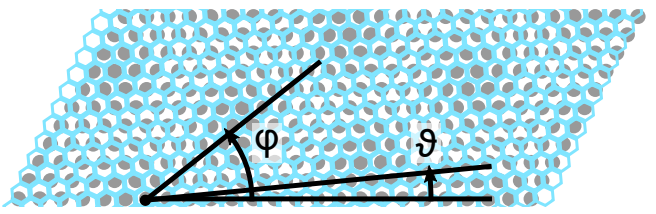

Figure 2: Overview and analysis of observed graphene domains on $\operatorname{Pt}(111)$ with indicated moiré spatial period $(\delta)$ and graphene orientation $(\vartheta)$. STM images of MLG domains with (a) $\delta=2.4 \mathrm{~nm}, \vartheta=4.8^{\circ}\left(100 \mathrm{mV}, 100 \mathrm{pA}, 8 \times 8 \mathrm{~nm}^{2}\right)$ and $(\mathrm{b}) \delta=0.8 \mathrm{~nm}, \vartheta=16.7^{\circ}(39 \mathrm{mV}$, $9 \mathrm{nA}, 8 \times 8 \mathrm{~nm}^{2}$ ). (c) MLG domain after the final annealing step with $\delta=0.8 \mathrm{~nm}, \vartheta=-15.8^{\circ}$ $\left(50 \mathrm{mV}, 100 \mathrm{nA}, 5 \times 5 \mathrm{~nm}^{2}\right)$. The dark depressions are attributed to $\mathrm{C}$ atoms or vacancies in the Pt surface. (d) Adjacent BLG and TLG domains $\left(400 \mathrm{mV}, 100 \mathrm{pA}, 20 \times 20 \mathrm{~nm}^{2}\right)$. TLG crosses a substrate step edge and ends approximately at the boundary marked by the dashed line. (e) Summary of $\delta, \vartheta$ for all observed moiré structures in MLG and BLG. The solid line depicts the expected variation of $\delta$ with $\vartheta$ for unstrained MLG. The upper (lower) dashed curve depicts $\delta$ as a function of $\vartheta$ for MLG with lattice constant increased (decreased) by $2 \%$. Open triangles (squares) are data for MLG after the first (second) CVD process, denoted as

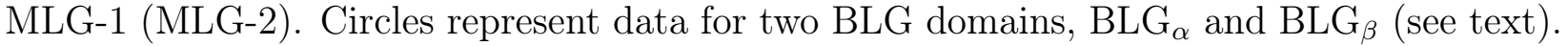
(f) Solid lines mark crystallographic directions of $\mathrm{Pt}(111)$ (dots), graphene (circles) and the resulting moiré superlattice. The orientations of graphene $(\vartheta)$ and moiré lattice $(\varphi)$ with respect to $\mathrm{Pt}(111)$ are indicated.

and dashed lines) are summarised in Fig. 2e. MLG data (triangles, squares) are reasonably well captured by the expected variation of $\delta$ with $\vartheta$ for unstrained MLG (Eq. S2). The dashed lines depict $\delta$ as a function of $\vartheta$ for a stretched (upper curve) and compressed (lower curve) $\mathrm{C}$ lattice, where the lattice constant deviates by $2 \%$ from the unstrained case. Deviations up to $7 \%$ were reported for stable configurations of MLG on $\operatorname{Pt}(111) .{ }^{35}$ Figure $2 \mathrm{f}$ defines the angles enclosed by crystallographic directions of the moiré superlattice and graphene with respect to $\mathrm{Pt}(111)$.

Some data, however, deviate significantly from the expected variation; that is, in an 

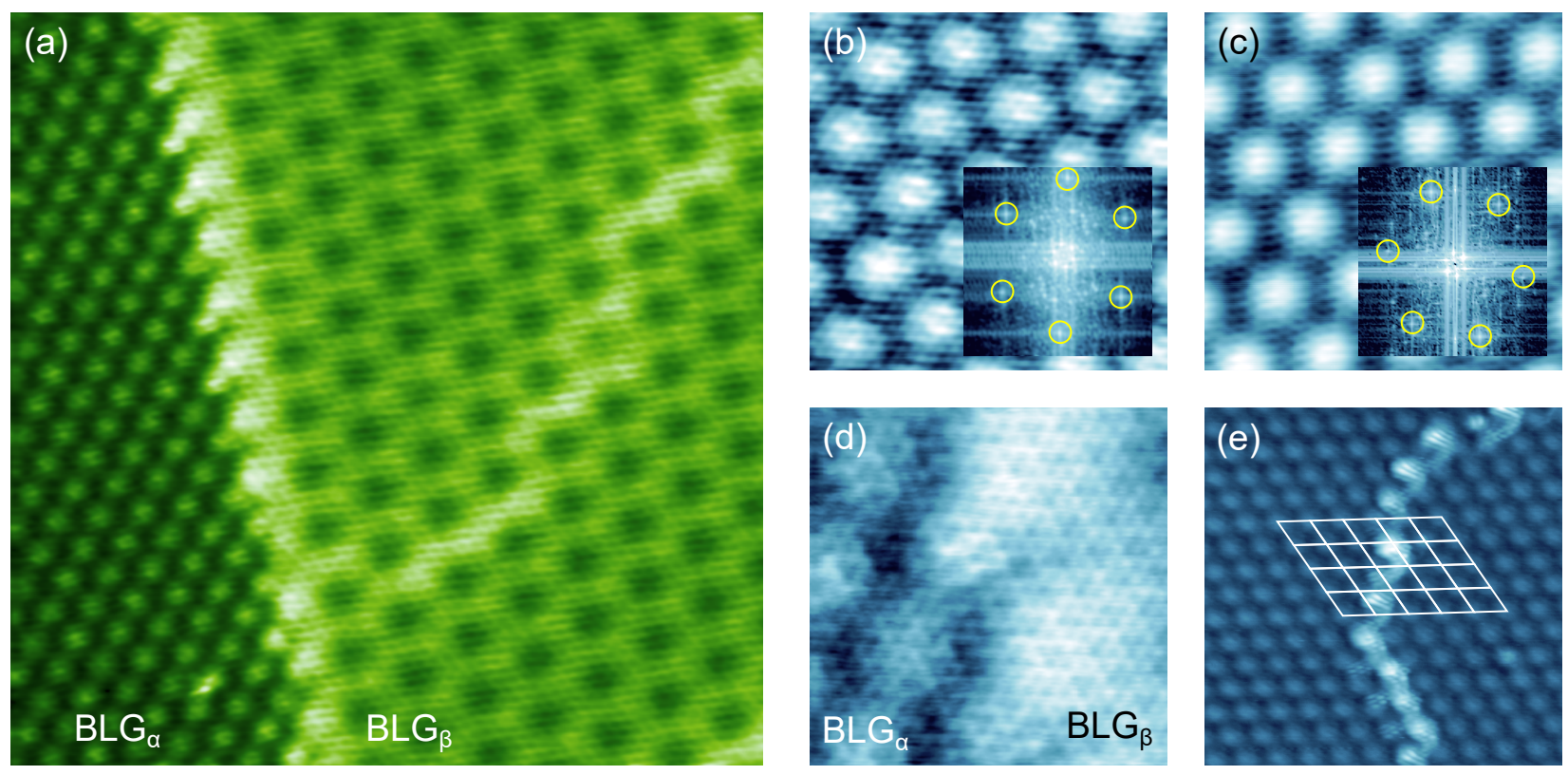

Figure 3: Graphene bilayer domains. (a) Overview STM image showing adjacent BLG ${ }_{\alpha}$ and BLG $_{\beta}$ domains $\left(70 \mathrm{mV}, 100 \mathrm{pA}, 30 \times 30 \mathrm{~nm}^{2}\right)$. (b), (c) STM images $\left(50 \mathrm{mV}, 7 \times 7 \mathrm{~nm}^{2}\right)$ of $\mathrm{BLG}_{\alpha}$ subdomains with identical moiré characteristics $\left(\delta=1.6 \mathrm{~nm}, \varphi=-28.8^{\circ}\right)$ and different graphene orientations, (b) $\vartheta=18.0^{\circ}$ and (c) $\vartheta=-18.0^{\circ}$. Tunnelling currents are $100 \mathrm{nA}$ (b) and $10 \mathrm{nA}$ (c). Insets: Fourier transforms of STM images with circles indicating the graphene lattice orientation. (d) Atomically resolved STM image of the transition from the $\mathrm{BLG}_{\alpha}\left(\delta=1.6 \mathrm{~nm}, \vartheta=18.0^{\circ}\right.$, left $)$ to the $\mathrm{BLG}_{\beta}$ moiré superlattice $\left(\delta=2.7 \mathrm{~nm}, \vartheta=18.0^{\circ}\right.$, right) $\left(70 \mathrm{mV}, 100 \mathrm{pA}, 5 \times 5 \mathrm{~nm}^{2}\right)$. (e) $\mathrm{STM}$ image of $\mathrm{BLG}_{\alpha}$ with a line defect in the top graphene layer $\left(1 \mathrm{~V}, 80 \mathrm{pA}, 20 \times 20 \mathrm{~nm}^{2}\right)$. The superimposed lattice shows that the moiré pattern is continuous across the line defect.

MLG model the observed moiré spatial period is not compatible with the observable $\vartheta$. The corresponding domains are only found after the second CVD process and are therefore assigned to BLG. The gallery of STM images in Fig. 3 further corroborates this assignment. Figure $3 \mathrm{a}$ shows an extended BLG region that contains both moiré patterns, $\mathrm{BLG}_{\alpha}(\delta=$ $\left.1.6 \mathrm{~nm}, \varphi=-28.8^{\circ}\right)$ and $\mathrm{BLG}_{\beta}\left(\delta=2.7 \mathrm{~nm}, \varphi=0.8^{\circ}\right) . \mathrm{BLG}_{\alpha}$ and $\mathrm{BLG}_{\beta}$ each exhibit two subdomains with identical $\delta$ and $\varphi$ but different $\vartheta$. Examplarily, this is demonstrated in Fig. 3b,c for the $\mathrm{BLG}_{\alpha}$ domain with atomic resolution. The Fourier transforms (insets to Fig. $3 \mathrm{~b}, \mathrm{c}$ ) reveal that the graphene lattices of the subdomains are rotated by $36^{\circ}$ with respect to each other, while the moiré pattern is unaffected. These observations unambiguously demonstrate the presence of two graphene sheets in $B L G_{\alpha}$ and $\mathrm{BLG}_{\beta}$ domains.

Remarkably, an atomically resolved close-up view of the domain boundary between BLG $_{\alpha}$ and $\mathrm{BLG}_{\beta}$ (Fig. 3d) reveals a unique graphene lattice covering both domains. Here, subdomains of $\mathrm{BLG}_{\alpha}$ and $\mathrm{BLG}_{\beta}$ with $\vartheta=18^{\circ}$ are connected. The uniformly oriented top layer of graphene demonstrates that the different moiré patterns must be due to rotational do- 
mains of the bottom graphene sheet. The STM image depicted in Fig. 3e corroborates this important aspect. It shows a $\mathrm{BLG}_{\alpha}$ area with a line defect in the top graphene sheet. The moiré pattern $\left(\delta=1.6 \mathrm{~nm}, \varphi=-28.8^{\circ}\right)$, however, remains unaffected across the defect as evidenced by the superimposed lattice. Therefore, the observed moire superstructure is due to the graphene/Pt(111) interface and, intriguingly, the upper graphene layer does not contribute to the moiré pattern.

Previously, moiré lattices on BLG/Ir(111) were attributed to the graphene/graphene interface. $^{20}$ The apparent contradiction with findings reported here is resolved when both the graphene/substrate and graphene/graphene twist angles are considered. For both these interfaces, low twist angles $\left(<10^{\circ}\right)$ give rise to an elevated hybridization, while large twist angles $\left(>10^{\circ}\right)$ decouple the layers effectively. ${ }^{20,39-41}$ In $\mathrm{BLG}_{\alpha}$ and $\mathrm{BLG}_{\beta}$ domains the bottom graphene layer is rotated by only $4.6^{\circ}$ and $0.1^{\circ}$ with respect to the $\mathrm{Pt}(111)$ surface, respectively (Eq. S4), while all graphene/graphene twist angles exceed $10^{\circ}$. In contrast, most of the reported domains of BLG/Ir(111) exhibit large twist angles at both interfaces. ${ }^{20}$ Consequently, the elevated (low) graphene-Pt (graphene-Ir) hybridization favours the observation of the moiré pattern due to the graphene/Pt (graphene/graphene) interface. The twist angles at both interfaces and the resulting interlayer coupling therefore determine which moiré superstructure is visible in STM images.

\section{Conclusions}

In conclusion, a preparation protocol for the preparation of extended BLG on $\mathrm{Pt}(111)$ has been introduced. The applicability of the method to other transition metal substrates and homogeneous as well as heterogeneous multistackings of two-dimensional materials may be anticipated. The presented atomically resolved STM data unveil that the moiré superlattices observed in BLG regions result from the graphene/Pt(111) interface.

\section{Experimental methods}

$\mathrm{Pt}(111)$ surfaces were prepared by $\mathrm{Ar}^{+}$bombardment and annealing at $1200 \mathrm{~K}$ in $\mathrm{O}_{2}$ atmosphere $\left(4 \cdot 10^{-5} \mathrm{~Pa}\right) \cdot \mathrm{C}_{2} \mathrm{H}_{4}$ at a partial pressure of $4 \cdot 10^{-5} \mathrm{~Pa}$ was used as a molecular precursor in the CVD process. After MLG growth via thermal decomposition of $\mathrm{C}_{2} \mathrm{H}_{4}$ at $1200 \mathrm{~K}$, Pt was evaporated from a hot filament at a rate of $\approx 1 \mathrm{ML} / \mathrm{min}$ (ML: monolayer), which was calibrated from STM images of several independent depositions on $\mathrm{Au}(100)$ and $\mathrm{MLG} / \mathrm{Pt}(111)$. The preparation of the second graphene layer was performed by annealing the sample at $1000 \mathrm{~K}$ for $3 \mathrm{~min}$, directly followed by exposure to $\mathrm{C}_{2} \mathrm{H}_{4}$ for $4 \mathrm{~min}$. The sample was kept at $1000 \mathrm{~K}$ for $5 \mathrm{~min}$ before cooling to room temperature. Additional annealing cycles were performed at $1200 \mathrm{~K}$. The experiments were performed in ultrahigh vacuum (base pressure $1 \cdot 10^{-8} \mathrm{~Pa}$ ) with a low-temperature STM operated at $6 \mathrm{~K}$. Tips were cut from Au wire and cleaned in situ by annealing and field emission. STM images were recorded in the constant-current mode with the bias voltage applied to the sample. Topographic data were processed using the WSxM software. ${ }^{42}$ 


\section{Conflicts of interest}

There are no conflicts to declare.

\section{Acknowledgements}

Financial support by the Deutsche Forschungsgemeinschaft through Grants No. KR 2912/101 and KR 2912/12 - 1 is acknowledged.

\section{References}

[1] E. McCann and M. Koshino, "The electronic properties of bilayer graphene," Rep. Prog. Phys., vol. 76, no. 5, p. 056503, 2013.

[2] K. S. Kim, A. L. Walter, L. Moreschini, T. Seyller, K. Horn, E. Rotenberg, and A. Bostwick, "Coexisting massive and massless dirac fermions in symmetry-broken bilayer graphene," Nat. Mater., vol. 12, no. 10, p. 887, 2013.

[3] D. V. Badami, "X-ray studies of graphite formed by decomposing silicon carbide," Carbon, vol. 3, no. 1, pp. $53-57,1965$.

[4] A. Van Bommel, J. Crombeen, and A. Van Tooren, "Leed and auger electron observations of the sic (0001) surface," Surf. Sci., vol. 48, no. 2, pp. 463-472, 1975.

[5] C. Riedl, C. Coletti, and U. Starke, "Structural and electronic properties of epitaxial graphene on sic $\left(\begin{array}{llll}0 & 0 & 0 & 1\end{array}\right)$ : a review of growth, characterization, transfer doping and hydrogen intercalation," J. Phys. D: Appl. Phys., vol. 43, no. 37, p. 374009, 2010.

[6] C. Oshima and A. Nagashima, "Ultra-thin epitaxial films of graphite and hexagonal boron nitride on solid surfaces," J. Phys.: Condens. Matter, vol. 9, no. 1, p. 1, 1997.

[7] J. Wintterlin and M.-L. Bocquet, "Graphene on metal surfaces," Surf. Sci., vol. 603, no. 10, pp. $1841-1852,2009$.

[8] J. Coraux, A. T. N'Diaye, M. Engler, C. Busse, D. Wall, N. Buckanie, F.-J. M. zu Heringdorf, R. van Gastel, B. Poelsema, and T. Michely, "Growth of graphene on ir(111)," New J. Phys., vol. 11, no. 2, p. 023006, 2009.

[9] J. Coraux, A. T. N'Diaye, C. Busse, and T. Michely, "Structural coherency of graphene on ir(111)," Nano Lett., vol. 8, no. 2, pp. 565-570, 2008.

[10] H. Hattab, A. T. N'Diaye, D. Wall, G. Jnawali, J. Coraux, C. Busse, R. van Gastel, B. Poelsema, T. Michely, F.-J. M. zu Heringdorf, and M. H. von Hoegen, "Growth temperature dependent graphene alignment on ir(111)," Appl. Phys. Lett., vol. 98, no. 14, p. 141903, 2011. 
[11] P. W. Sutter, J.-I. Flege, and E. A. Sutter, "Epitaxial graphene on ruthenium," Nat. Mater., vol. 7, no. 5, p. 406, 2008.

[12] A. Reina, X. Jia, J. Ho, D. Nezich, H. Son, V. Bulovic, M. S. Dresselhaus, and J. Kong, "Large area, few-layer graphene films on arbitrary substrates by chemical vapor deposition," Nano Lett., vol. 9, no. 1, pp. 30-35, 2009.

[13] Y. Cui, Q. Fu, and X. Bao, "Dynamic observation of layer-by-layer growth and removal of graphene on ru(0001)," Phys. Chem. Chem. Phys., vol. 12, pp. 5053-5057, 2010.

[14] J.-H. Gao, D. Fujita, M.-S. Xu, K. Onishi, and S. Miyamoto, "Unique synthesis of few-layer graphene films on carbon-doped pt83rh17 surfaces," ACS Nano, vol. 4, no. 2, pp. 1026-1032, 2010.

[15] M. Xu, D. Fujita, K. Sagisaka, E. Watanabe, and N. Hanagata, "Production of extended single-layer graphene," ACS Nano, vol. 5, no. 2, pp. 1522-1528, 2011.

[16] E. Zhizhin, D. Pudikov, A. Rybkin, A. Petukhov, Y. Zhukov, and A. Shikin, "Growth of graphene monolayer by "internal solid-state carbon source": Electronic structure, morphology and au intercalation," Mater. Des., vol. 104, pp. 284 - 291, 2016.

[17] D. Pudikov, E. Zhizhin, A. Rybkin, and A. Shikin, "Graphene fabrication via carbon segregation through transition metal films," Thin Solid Films, vol. 648, pp. 120 - 127, 2018.

[18] S. Nie, A. L. Walter, N. C. Bartelt, E. Starodub, A. Bostwick, E. Rotenberg, and K. F. McCarty, "Growth from below: Graphene bilayers on ir(111)," ACS Nano, vol. 5, no. 3, pp. 2298-2306, 2011.

[19] C. Herbig, T. Knispel, S. Simon, U. A. Schröder, A. J. Martínez-Galera, M. A. Arman, C. Teichert, J. Knudsen, A. V. Krasheninnikov, and T. Michely, "From permeation to cluster arrays: Graphene on ir(111) exposed to carbon vapor," Nano Lett., vol. 17, no. 5, pp. 3105-3112, 2017.

[20] S. Simon, E. Voloshina, J. Tesch, F. Förschner, V. Enenkel, C. Herbig, T. Knispel, A. Tries, J. Kröger, Y. Dedkov, and M. Fonin, "Layer-by-layer decoupling of twisted graphene sheets epitaxially grown on a metal substrate," Small, vol. 14, no. 13, p. 1703701, 2018.

[21] C. Riedl, C. Coletti, T. Iwasaki, A. A. Zakharov, and U. Starke, "Quasi-free-standing epitaxial graphene on sic obtained by hydrogen intercalation," Phys. Rev. Lett., vol. 103, p. 246804, Dec 2009.

[22] C. Enderlein, Y. S. Kim, A. Bostwick, E. Rotenberg, and K. Horn, "The formation of an energy gap in graphene on ruthenium by controlling the interface," New Journal of Physics, vol. 12, no. 3, p. 033014, 2010. 
[23] P. Leicht, L. Zielke, S. Bouvron, R. Moroni, E. Voloshina, L. Hammerschmidt, Y. S. Dedkov, and M. Fonin, "In situ fabrication of quasi-free-standing epitaxial graphene nanoflakes on gold," ACS Nano, vol. 8, no. 4, pp. 3735-3742, 2014.

[24] M. Gyamfi, T. Eelbo, M. Waśniowska, and R. Wiesendanger, "Impact of intercalated cobalt on the electronic properties of graphene on pt(111)," Phys. Rev. B, vol. 85, p. 205434, May 2012.

[25] R. Decker, J. Brede, N. Atodiresei, V. Caciuc, S. Blügel, and R. Wiesendanger, "Atomicscale magnetism of cobalt-intercalated graphene," Phys. Rev. B, vol. 87, p. 041403, Jan 2013.

[26] M. Sicot, P. Leicht, A. Zusan, S. Bouvron, O. Zander, M. Weser, Y. S. Dedkov, K. Horn, and M. Fonin, "Size-selected epitaxial nanoislands underneath graphene moiré on rh(111)," ACS Nano, vol. 6, no. 1, pp. 151-158, 2012. PMID: 22214768.

[27] D. Pacilé, P. Leicht, M. Papagno, P. M. Sheverdyaeva, P. Moras, C. Carbone, K. Krausert, L. Zielke, M. Fonin, Y. S. Dedkov, F. Mittendorfer, J. Doppler, A. Garhofer, and J. Redinger, "Artificially lattice-mismatched graphene/metal interface: Graphene/ni/ir(111)," Phys. Rev. B, vol. 87, p. 035420, Jan 2013.

[28] M. Papagno, P. Moras, P. M. Sheverdyaeva, J. Doppler, A. Garhofer, F. Mittendorfer, J. Redinger, and C. Carbone, "Hybridization of graphene and a ag monolayer supported on re(0001)," Phys. Rev. B, vol. 88, p. 235430, Dec 2013.

[29] S. Schumacher, T. O. Wehling, P. Lazić, S. Runte, D. F. Förster, C. Busse, M. Petrović, M. Kralj, S. Blügel, N. Atodiresei, V. Caciuc, and T. Michely, "The backside of graphene: Manipulating adsorption by intercalation," Nano Lett., vol. 13, no. 11, pp. 5013-5019, 2013.

[30] M. Petrović, I. Šrut Rakić, S. Runte, C. Busse, J. T. Sadowski, P. Lazić, I. Pletikosić, Z.H. Pan, M. Milun, P. Pervan, N. Atodiresei, R. Brako, D. Šokčević, T. Valla, T. Michely, and M. Kralj, "The mechanism of caesium intercalation of graphene," Nat. Commun., vol. 4, p. 2772, 2013.

[31] J. Halle, N. Néel, and J. Kröger, "Filling the gap: Li-intercalated graphene on ir(111)," The Journal of Physical Chemistry C, vol. 120, no. 9, pp. 5067-5073, 2016.

[32] A. B. Preobrajenski, M. L. Ng, A. S. Vinogradov, and N. Mårtensson, "Controlling graphene corrugation on lattice-mismatched substrates," Phys. Rev. B, vol. 78, p. 073401, Aug 2008.

[33] M. Gao, Y. Pan, L. Huang, H. Hu, L. Z. Zhang, H. M. Guo, S. X. Du, and H.-J. Gao, "Epitaxial growth and structural property of graphene on pt(111)," Applied Physics Letters, vol. 98, no. 3, p. 033101, 2011. 
[34] Z. Liang, H. Khosravian, A. Uhl, R. J. Meyer, and M. Trenary, "Graphene domain boundaries on pt(111) as nucleation sites for pt nanocluster formation," Surf. Sci., vol. 606, no. 21 , pp. 1643 - 1648, 2012.

[35] P. Merino, M. Švec, A. L. Pinardi, G. Otero, and J. A. Martín-Gago, "Strain-driven moiré superstructures of epitaxial graphene on transition metal surfaces," ACS Nano, vol. 5, no. 7, pp. 5627-5634, 2011.

[36] L. Jin, Q. Fu, Y. Yang, and X. Bao, "A comparative study of intercalation mechanism at graphene/ru(0001) interface," Surf. Sci., vol. 617, pp. 81 - 86, 2013.

[37] X. Feng, S. Maier, and M. Salmeron, "Water splits epitaxial graphene and intercalates," J. Am. Chem. Soc., vol. 134, no. 12, pp. 5662-5668, 2012.

[38] G. Otero, C. González, A. L. Pinardi, P. Merino, S. Gardonio, S. Lizzit, M. Blanco-Rey, K. Van de Ruit, C. F. J. Flipse, J. Méndez, P. L. de Andrés, and J. A. Martín-Gago, "Ordered vacancy network induced by the growth of epitaxial graphene on pt(111)," Phys. Rev. Lett., vol. 105, p. 216102, Nov 2010.

[39] J. Hass, F. Varchon, J. E. Millán-Otoya, M. Sprinkle, N. Sharma, W. A. de Heer, C. Berger, P. N. First, L. Magaud, and E. H. Conrad, "Why multilayer graphene on $4 h-\mathrm{SiC}(000 \overline{1})$ behaves like a single sheet of graphene," Phys. Rev. Lett., vol. 100, p. 125504, Mar 2008.

[40] A. Luican, G. Li, A. Reina, J. Kong, R. R. Nair, K. S. Novoselov, A. K. Geim, and E. Y. Andrei, "Single-layer behavior and its breakdown in twisted graphene layers," Phys. Rev. Lett., vol. 106, p. 126802, Mar 2011.

[41] E. Starodub, A. Bostwick, L. Moreschini, S. Nie, F. E. Gabaly, K. F. McCarty, and E. Rotenberg, "In-plane orientation effects on the electronic structure, stability, and raman scattering of monolayer graphene on ir(111)," Phys. Rev. B, vol. 83, p. 125428, Mar 2011.

[42] I. Horcas, R. Fernández, J. Gomez-Rodriguez, J. Colchero, J. Gómez-Herrero, and A. Baro, "Wsxm: a software for scanning probe microscopy and a tool for nanotechnology," Rev. Sci. Instrum., vol. 78, no. 1, p. 013705, 2007. 


\title{
Supplementary information \\ Preparation of graphene bilayers on platinum by sequential chemical vapor deposition
}

\author{
Johannes Halle, ${ }^{*}$ Alexander Mehler, Nicolas Néel, Jörg Kröger \\ Institut für Physik, Technische Universität Ilmenau, D-98693 Ilmenau, Germany \\ E-mail: johannes.halle@tu-ilmenau.de
}

\section{Intercalation of deposited Pt film}

In order to further evidence that the buried monolayer graphene (MLG) forms the bottom layer in bilayer graphene (BLG), Fig. S1 demonstrates the entire intercalation of the thick Pt film through the buried MLG. Upon annealing the Pt-covered MLG/Pt(111) sample (Fig. S1a) at $1200 \mathrm{~K}$, the deposited Pt is flattening and intercalating under MLG (Fig. S1b,c). The segregated MLG exhbits the characteristic moiré pattern (Fig. S1d).
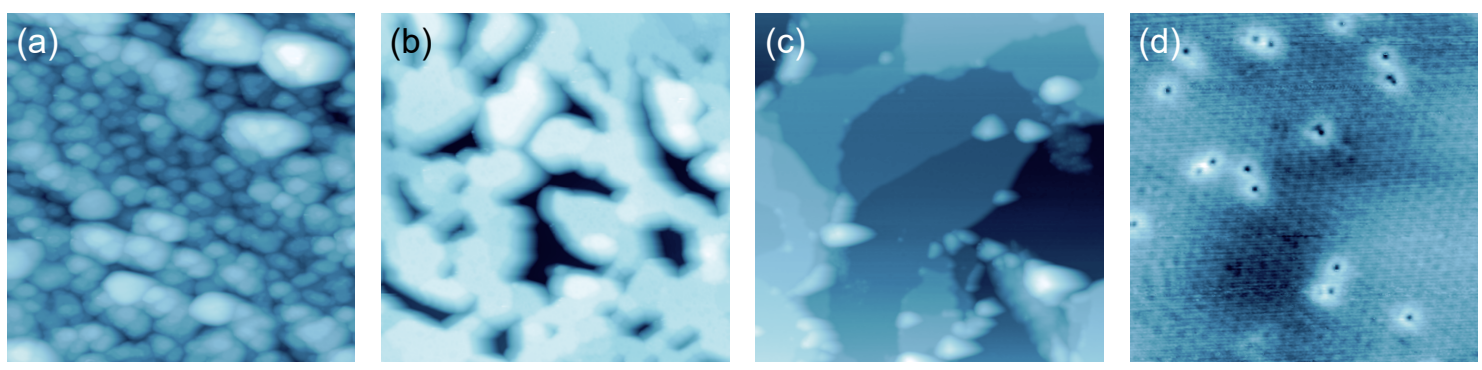

Figure S1: Pt intercalation of MLG-covered Pt(111). (a) STM image of Pt film deposited on MLG/Pt(111) $\left(1 \mathrm{~V}, 80 \mathrm{pA}, 120 \times 120 \mathrm{~nm}^{2}\right)$. (b) STM image of the deposited Pt smoothed by annealing at $720 \mathrm{~K}\left(1.5 \mathrm{~V}, 80 \mathrm{pA}, 150 \times 150 \mathrm{~nm}^{2}\right)$. (c) STM image of MLG/Pt(111) after complete intercalation of the deposited Pt at $1200 \mathrm{~K}\left(1.5 \mathrm{~V}, 80 \mathrm{pA}, 100 \times 100 \mathrm{~nm}^{2}\right)$. (d) MLG moiré pattern observed on the sample shown in $(\mathrm{c})\left(0.1 \mathrm{~V}, 100 \mathrm{pA}, 20 \times 20 \mathrm{~nm}^{2}\right)$. 


\section{Analysis of graphene moiré patterns}

\section{Moiré patterns of monolayer graphene on $\mathrm{Pt}(111)$}

The moiré pattern is generated by the superposition of $\mathrm{Pt}(111)$ and the monolayer graphene (MLG) lattices. In direct space, $a_{\mathrm{Pt}}, a_{\mathrm{C}}, \delta$ denote the spatial periods of, respectively, the $\operatorname{Pt}(111)$ surface, the graphene lattice, the moiré superstructure. In reciprocal space, the corresponding lattice vectors are $\mathbf{k}_{\mathrm{Pt}}, \mathbf{k}_{\mathrm{C}}, \mathbf{k}_{\mathrm{m}}$ with magnitudes $k_{\mathrm{Pt}}=\left|\mathbf{k}_{\mathrm{Pt}}\right|=1 / a_{\mathrm{Pt}}, k_{\mathrm{C}}=$ $\left|\mathbf{k}_{\mathrm{C}}\right|=1 / a_{\mathrm{C}}, k_{\mathrm{m}}=\left|\mathbf{k}_{\mathrm{m}}\right|=1 / \delta$. The moiré superstructure represents a spatial beating pattern, which may be expressed by ${ }^{1}$

$$
\mathbf{k}_{\mathrm{m}}=\mathbf{k}_{\mathrm{C}}-\mathbf{k}_{\mathrm{Pt}} .
$$

with $\vartheta=\angle\left(\mathbf{k}_{\mathrm{Pt}}, \mathbf{k}_{\mathrm{C}}\right)$ and $\varphi=\angle\left(\mathbf{k}_{\mathrm{Pt}}, \mathbf{k}_{\mathrm{m}}\right)$ defined as the smallest angles enclosed by the respective lattice orientations (Fig. S1). Due to the hexagonal symmetry of the lattices, $\vartheta$ and $\varphi$ are constrained to the interval $\left[-30^{\circ}, 30^{\circ}\right]$. Spatial periods and orientations of the moiré pattern may readily be derived from the triangle formed by the three reciprocal lattice vectors (Fig. S1a). Using the law of cosines yields $\delta$ as a function of $\vartheta$,

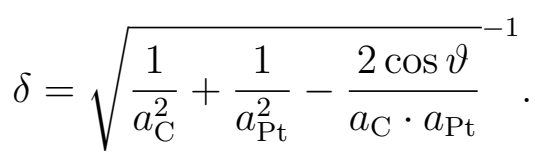

Possible strain in the graphene sheet can be included by scaling the graphene lattice constant appropriately. Fig. 2e of the manuscript shows the resulting $\delta$-versus- $\vartheta$ curves. Since $\delta$ is an even function of $\vartheta$, the interval $\vartheta=\left[0^{\circ}, 30^{\circ}\right]$ contains all possible moiré patterns.

\section{Characterization of the bottom graphene layer in bilayer graphene domains}

An important conclusion of the findings presented in the main manuscript is the origin of the moiré pattern observed from bilayer graphene (BLG) domains. The results evidence that the moiré superstructure is caused by the interface beween the bottom graphene layer and $\mathrm{Pt}(111)$. Using the moiré characteristics observed from BLG $\left(\mathrm{BLG}_{\alpha}, \mathrm{BLG}_{\beta}\right.$ in Fig. 2e), the spatial period and orientation of the bottom graphene layer may be deduced as follows. Given the spatial moiré period $\delta$ and its orientation $\varphi$ with respect to the $\operatorname{Pt}(111)$ lattice, six moiré orientations defined by $\varphi_{\text {mod }}$ in Fig. S1a are indistinguishable in the experiments. These orientations differ by $60^{\circ}$. Free parameters are the unknown orientation of the bottom graphene layer, $\vartheta_{\mathrm{b}}$, and its lattice constant, $a_{\mathrm{C}}$, which may be affected by strain. The solutions with minimal strain are considered most plausible and can be obtained by calculating $a_{\mathrm{C}}$ for every $\varphi_{\bmod }$,

$$
a_{\mathrm{C}}=\sqrt{\frac{1}{a_{\mathrm{Pt}}^{2}}+\frac{1}{\delta^{2}}+\frac{2 \cos \varphi_{\mathrm{mod}}}{a_{\mathrm{Pt}} \cdot \delta}} .
$$


(a)

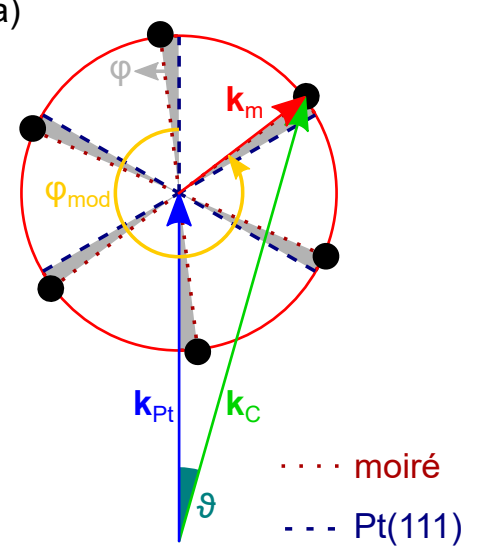

(b)

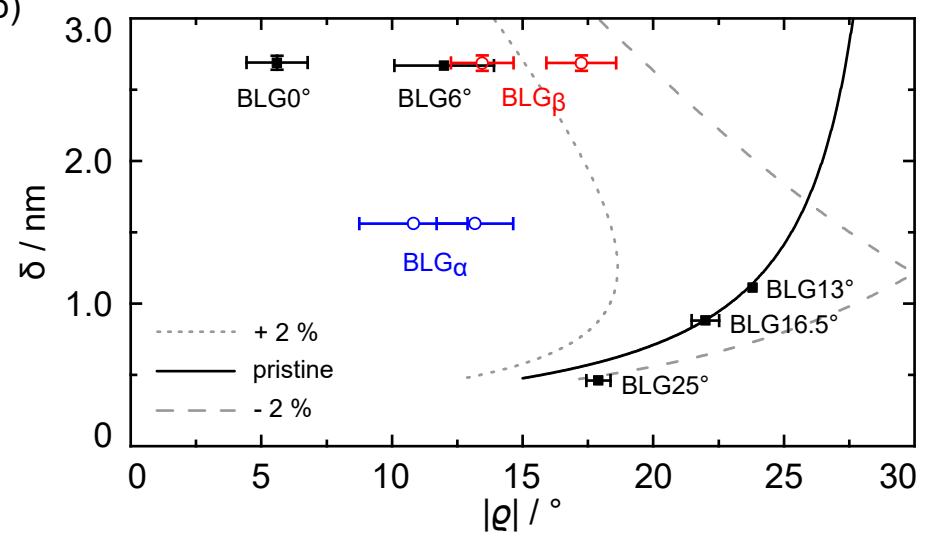

Figure S2: (a) Illustration of the moiré construction. The reciprocal lattice vectors of $\operatorname{Pt}(111)\left(\mathbf{k}_{\mathrm{Pt}}\right)$, graphene $\left(\mathbf{k}_{\mathrm{C}}\right)$ and the moiré pattern $\left(\mathbf{k}_{\mathrm{m}}\right)$ form a triangle. The angle enclosed by crystallographic directions of $\operatorname{Pt}(111)$ and graphene is denoted as $\vartheta=\angle\left(\mathbf{k}_{\mathrm{Pt}}, \mathbf{k}_{\mathrm{C}}\right)$, while $\varphi=L\left(\mathbf{k}_{\mathrm{Pt}}, \mathbf{k}_{\mathrm{m}}\right)$ is the angle between crystallographic directions of $\mathrm{Pt}(111)$ and the moiré lattice. Dashed and dotted lines represent symmetry directions of, respectively, $\operatorname{Pt}(111)$ and the moiré superstructure. Dots on the circle with radius $k_{\mathrm{m}}=\left|\mathbf{k}_{\mathrm{m}}\right|$ indicate equivalent moiré superstructures with $\varphi_{\bmod }=\varphi+n \cdot 60^{\circ}(n=1,2, \ldots, 5)$, which reproduce the same observable moiré characteristics for varying $\mathbf{k}_{\mathrm{C}}$. (b) Twisted bilayer graphene (BLG) model. Lines depict the calculated moiré spatial period $\delta$ as a function of the angle $\varrho$ enclosed by crystallographic directions of the upper graphene lattice and the moiré superstructure. The solid line shows the variation of $\delta$ with $|\varrho|$ for an unstrained lower graphene sheet, whereas dotted and dashed lines represent the situation for a graphene lattice constant that is increased and decreased by $2 \%$, respectively. Experimental data appear as circles (this work) and squares $(\mathrm{BLG} / \operatorname{Ir}(111))^{2}$. The angles $0^{\circ} \ldots 25^{\circ}$ denote the twist angles between the bottom and upper graphene sheets.

Subsequently, $\vartheta_{\mathrm{b}}$ is calculated for the selected $a_{\mathrm{C}}^{\min }$ with minimal strain at the angle $\varphi_{\bmod }^{\min }$ using

$$
\delta \sin \vartheta_{\mathrm{b}}=a_{\mathrm{C}}^{\min } \sin \varphi_{\bmod }^{\min }
$$

Following this procedure, the bottom graphene sheets of the observed BLG domains are characterized by $\vartheta_{\mathrm{b}}=4.6^{\circ}$ with $a_{\mathrm{C}}=240 \mathrm{pm}(-2.5 \%$ strain $)$ in the case of $\mathrm{BLG}_{\alpha}$ and $\vartheta_{\mathrm{b}}=0.1^{\circ}$ with $a_{\mathrm{C}}=252 \mathrm{pm}(2.1 \%$ strain $)$ in the $\mathrm{BLG}_{\beta}$ domain. Consequently, the twist angles $\theta$ between adjacent graphene layers may now be obtained as $\theta=22.6^{\circ}, 13.4^{\circ}$ for $\mathrm{BLG}_{\alpha}$ and $\theta=12.6^{\circ}, 18.1^{\circ}$ for $\mathrm{BLG}_{\beta}$ subdomains.

\section{Moiré patterns of twisted bilayer graphene}

The moiré pattern generated by twisted BLG can be calculated using Eq. S2 with $a_{\mathrm{Pt}}$ replaced by $a_{\mathrm{C}}$. Thus, the spatial period of a moiré pattern resulting from two unstrained graphene 
lattices is described by

$$
\delta=\frac{a_{\mathrm{C}}}{\sqrt{2(1-\cos \theta)}} .
$$

Assuming that the twist angle between bottom (b) and top (t) graphene sheets causes the observed moiré pattern, b-graphene may be characterized on the basis of this moiré pattern. To this end, t-graphene takes the role of the Pt lattice in Eqs. S3, S4 with known lattice constant $\mathrm{a}_{\mathrm{C}, \mathrm{t}}$ and as a reference lattice for all angles. The lattice constant $a_{\mathrm{C}, \mathrm{b}}$ of the lower graphene is described by

$$
a_{\mathrm{C}, \mathrm{b}}=\sqrt{\frac{1}{a_{\mathrm{C}, \mathrm{t}}^{2}}+\frac{1}{\delta^{2}}+\frac{2 \cos \varrho_{\mathrm{mod}}}{a_{\mathrm{C}, \mathrm{t}} \cdot \delta}}
$$

for each of the six possible orientations defined by $\varrho_{\text {mod }}$, which describes the angle enclosed by the moiré superstructure and t-graphene. The twist angle $\theta$ between the two graphene sheets is then given by

$$
\delta \sin \theta=a_{\mathrm{C}, \mathrm{b}}^{\min } \sin \varrho_{\bmod }^{\min } .
$$

Fig. S1b shows BLG data obtained in this work as well as from a recent study of BLG on $\operatorname{Ir}(111)$, where the observed moiré patterns were attributed to twisted graphene bilayers ${ }^{2}$. While data for larger twist angles $\left(>13^{\circ}\right)$ follow the expected behavior for pristine BLG reasonably well, there is a notable deviation at smaller rotation angles. Indeed, the BLG0 ${ }^{\circ}$ moiré superstructure is virtually that of the underlying graphene/substrate interface. Density functional calculations ${ }^{2}$ showed that low twist angles give rise to an elevated graphene-metal coupling, which is consistent with the mechanism discussed in the main manuscript. In the twisted-BLG model, the $\mathrm{BLG}_{\alpha}$ subdomains (Fig. 3c,d) would require comparably large strains of $3.5 \%$ and $4.2 \%$, but the $\mathrm{BLG}_{\beta}$ subdomains would exhibit only $1.6 \%$ and $2.3 \%$ strain. However, the resulting moiré patterns of the subdomains would have to align in order to reproduce the experimental observations. Therefore, the twisted-BLG model is not appropriate to explain the observed moiré structures.

\section{References}

[1] A. T. N'Diaye, J. Coraux, T. N. Plasa, C. Busse, and T. Michely, "Structure of epitaxial graphene on $\operatorname{ir}(111), "$ New J. Phys., vol. 10, no. 4, p. 043033, 2008.

[2] S. Simon, E. Voloshina, J. Tesch, F. Förschner, V. Enenkel, C. Herbig, T. Knispel, A. Tries, J. Kröger, Y. Dedkov, and M. Fonin, "Layer-by-layer decoupling of twisted graphene sheets epitaxially grown on a metal substrate," Small, vol. 14, no. 13, p. 1703701, 2018. 